\title{
Variants of Green Fluorescent Protein GFPxm
}

\author{
Wen-Xin Luo, Tong Cheng, Bao-Quan Guan, Shao-Wei Li, Ji Miao, Jun Zhang, \\ Ning-Shao Xia
}

National Institute of Diagnostics and Vaccine Development in infectious disease, The Key Laboratory of Ministry of Education for Cell Biology and Tumor Cell Engineering, Xiamen University, Xiamen, 361005, Peoples' Republic of China

Received: 6 January 2006 / Accepted: 26 March 2006 / Published online 3 July 2006

\section{Abstract}

As research progresses, fluorescent proteins useful for optical marking will evolve toward brighter, monomeric forms that are more diverse in color. We previously reported a new fluorescent protein from Aequorea macrodactyla, GFPxm, that exhibited many characteristics similar to wild-type green fluorescent protein (GFP). However, the application of GFPxm was limited because GFPxm expressed and produced fluorescence only at low temperatures. To improve the fluorescent properties of GFPxm, 12 variants were produced by site-directed mutagenesis and DNA shuffling. Seven of these mutants could produce strong fluorescence when expressed at $37^{\circ} \mathrm{C}$. The relative fluorescence intensities of mutants GFPxm16, GFPxm18, and GFPxm19 were higher than that of EGFP (enhanced GFP) when the expression temperature was between 25 and $37^{\circ} \mathrm{C}$, and mutants GFPxm16 and GFPxm163 could maintain a high fluorescence intensity even when expressed at $42^{\circ} \mathrm{C}$. Meanwhile, at least 4 mutants could be successfully expressed in mammalian cell lines. The fluorescence spectra of 6 of the 12 mutants had a progressive red shift. The longest excitation-emission maximum was at $514 / 525 \mathrm{~nm}$. In addition, 3 of the 12 mutants had two excitation peaks including an UV-excitation peak, while another mutant had only one UVexcitation peak.

Keywords: Fluorescence spectra - green fluorescent protein - variants

\section{Introduction}

Green fluorescent protein (GFP) from the jellyfish Aequorea victoria and its variants have been used to

Correspondence to: Ning-Shao Xia; E-mail: nsxia@xmu.edu.cn track and quantify individual or multiple protein species, monitor protein-protein interactions, and describe biological events. The application of GFP has revolutionized many areas of cell biology and biotechnology (Lippincott-Schwartz and Patterson, 2003).

To expand the use of fluorescent probes, a great deal of effort is directed to engineering new reporter classes with novel fluorescent properties. More than 100 GFP homologs have been obtained from nonbioluminescent Anthozoa species since 1999. These proteins exhibit color diversity that includes green, yellow, and red fluorescence, as well as purple-blue nonfluorescence (Labas et al., 2002). Generation of photoactivatable and photoswitchable derivatives enabled researchers to selectively activate individual molecules or groups of molecules and easily track their behavior in vivo (Ando et al., 2002; Chudakov et al., 2003, 2004; Verkhusha and Sorkin, 2005). Dronpa, a GFP-like protein with sensitive and reversible on-off switching capabilities, represents a new generation of specialized fluorescent tools for studying protein movement and trafficking inside cells (Ando et al., 2004).

Although the aforementioned GFP-like proteins from nonbioluminescent Anthozoa are useful as fluorescent labels, their application is limited by their oligomeric state. These proteins are tetramers, which poses a problem when they are used as fusion proteins with other oligomeric proteins, while GFPs from jellyfish are fluorescent as $28-\mathrm{kDa}$ monomers. Attempts have been made to produce their monomeric derivatives (Campbell et al., 2002; Karasawa et al., 2003; Shaner et al., 2004). However, the extinction coefficients, quantum yields, or photostabilities of some are currently far from optimal.

It is important to obtain fluorescent proteins that are bright, monomeric, photoconvertable with high efficiency and specificity, and diverse in color. We report here engineering studies of a GFP-like protein from Aequorea macrodactyla and show that 
the new variants can be produced with distinct fluorescent properties and are applicable for imaging of mammalian cells.

\section{Materials and Methods}

Enzymes and Chemicals. Fluorescein isothiocyanate (FITC), T4 ligase, and restriction endonucleases were purchased from Promega (Madison, WI). A gel extraction kit was purchased from Watson (Shanghai, China). Taq polymerase and dNTPs were purchased from Sangon (Shanghai, China). DNase I was purchased from Takara (Kyoto, Japan). LipofectAMINE ${ }^{\mathrm{TM}} 2000$ transfection reagent was purchased from Invitrogen (Carlsbad, CA).

Bacterial Strains and Plasmids. E.coli strain ER2566 was purchased from Promega. Plasmids pEGFP, pGFPuv, pEYFP, and pEBFP were purchased from Clontech Laboratories (Palo Alto, CA), while pTO-T7, pTO-T7EGFP, and pTO-T7GFPxm were constructed in our laboratory as described previously (Luo et al., 2000; Xia et al., 2002).

Site-Directed Mutagenesis and Protein Expression. The encoding cDNA was cloned into the BamHI/HindIII site of expression vector pTO-T7. Site-directed mutagenesis was performed via polymerase chain reaction (PCR) using the overlap extension method, with primers containing appropriate target substitutions. The $5^{\prime}$ primer T7F matched the $\mathrm{T} 7$ promoter sequence of the vector. The $3^{\prime}$ primer T7T was complementary to the $\mathrm{T} 7$ terminator sequence of the vector. Appropriate mutagenic primers (sense) that partially matched the gfps sequences were designed, and mutations were introduced where the nucleotide should be replaced. The other $3^{\prime}$ primers (antisense) were completely complementary to the $5^{\prime}$ of the mutagenic primers. The amino acids for the replacement of F64, S65, Q69, and T203 of GFPxm are: F or L (F64); A, G, T, or S (S65); L, Q, or K (Q69); and Y, C, F, H, R, L, Q, or $\mathrm{W}$ (T203). The amplified fragments were pooled, digested with BamHI and HindIII, and then ligated to the pTO-T7 vector cleaved with the same enzymes. The ligation mixture was transformed into the bacterial strain ER2566. Colonies were selected and then incubated at $37^{\circ} \mathrm{C}$ in $3 \mathrm{ml}$ of $\mathrm{LB}$ medium $(100 \mu \mathrm{g} / \mathrm{ml}$ of kanamycin) until reaching an optical density of 0.8 at $600 \mathrm{~nm}$. Isopropyl- $\beta$-d-thiogalactopyramoside (IPTG) was added to a final concentration of $0.2 \mathrm{mmol} / \mathrm{L}$, and the cultures were further incubated at $25^{\circ} \mathrm{C}$ for $6 \mathrm{~h}$. Excitation and emission spectrum of various expressed GFPxm mutants were measured by fluorescent panorama (Fluorat-
02 Panorama Lumaex, Russia). The sequences were verified by DNA sequencing at Bioasia (Shanghai, China).

DNA Shuffling. The encoding sequences for fluorescent proteins in pEGFP, pEYFP, pGFPuv, and pEBFP were subcloned into the BamHI/HindIII site of pTO-T7 to produce pTO-T7EGFP, pTO-T7EYFP, pTO-T7GFPuv, and pTO-T7EBFP. Equal amounts of these plasmids and those of pTO-T7GFPxm, pTO-T7GFPxm16, pTO-T7GFPxm18, and pTOT7GFPxm19 (described in the Results and Table 1) were mixed as the templates in PCR with T7F/T7R primers. The resulting DNA fragments of about $850 \mathrm{bp}$ were purified and used for DNA shuffling (Crameri et al., 1996). The library of in vitro-recombined GFP genes were recloned in the pTO-T7 vector at the BamH I/HindIII sites and transformed to ER2566.

Effects of Culture Temperature and Time on the Expression Level and the Fluorescent Intensity of GFPxm Mutants. ER2566 cells containing various constructs of GFPxm mutants were grown to an optical density of 0.25 at $600 \mathrm{~nm}$ and then induced with $0.2 \mathrm{mM}$ IPTG at 25,32 , and $42^{\circ} \mathrm{C}$ for $6 \mathrm{~h}$, or at $37^{\circ} \mathrm{C}$ for $10 \mathrm{~h}$. When induced in $37^{\circ} \mathrm{C}$, samples were withdrawn at indicated times. For each sample, the fluorescence of an equal optical density of 0.225 at $600 \mathrm{~nm}$ was measured. F-4500 Hitachi fluorescence spectrophotometer was used for spectral measurements. The excitation and emission slits were set at $5 \mathrm{~nm}$. The amounts of intracellular recombinant protein were determined by quantification of band intensities by UVIBAND software for windows (version 99) and the total bacterial proteins were regarded as 100 . Fluorescence values were then normalized against the amount of recombinant protein present in the cell to give a relative measure of the proportion of intracellular GFP that is fluorescent for each culture.

Purification and Analysis of Various Recombinant GFPxm Mutants. The purification and the analysis of fluorescence properties of the expressed GFPxm mutants were performed according to the methods described previously (Xia et al., 2002).

Expression of GFPxm Mutants in Mammalian Cells. DNA fragments of GFPxm16, GFPxm18, GFPxm19, GFPxm163, GFPxm19uv, and OFPxm were inserted into expression vector pcDNA3.1 and digested with BamHI and XhoI. Resultant plasmids were transfected into $\mathrm{CHO}$, Hela, and 
Table 1. Fluorescent Characteristics of GFPxm and Its Mutants

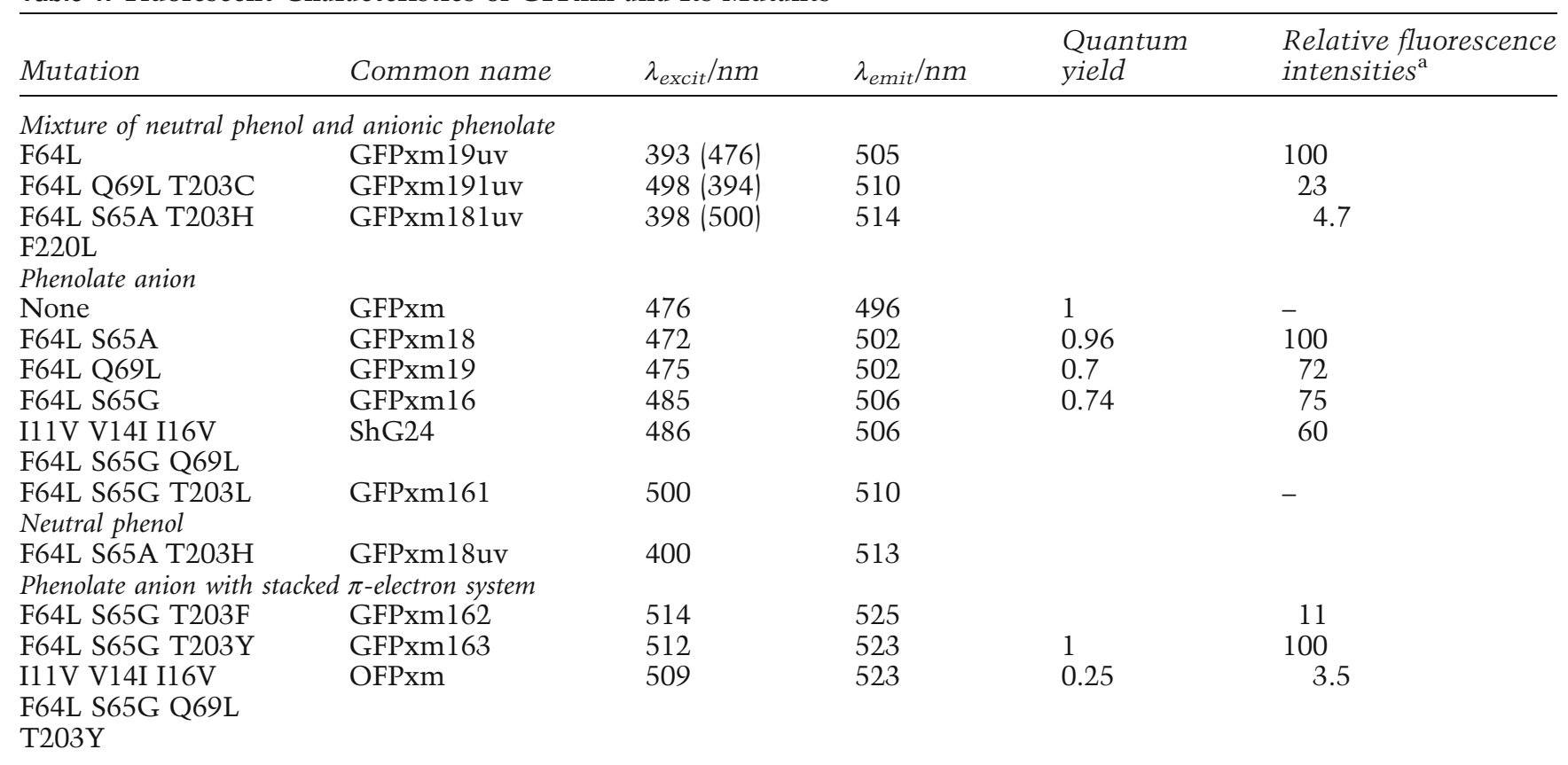

${ }^{a}$ Expression results at $37^{\circ} \mathrm{C}$. They have been arbitrarily normalized to 100 for the brightest member of each class and cannot be used to compare different classes. The fluorescent intensity measurements of the first class were under the condition of UV excitement. -, Extremely low. The other parameters were not detected.

HepG2 cells according to the manufacturer's instructions for LipofecTAMINE ${ }^{\mathrm{TM}}$ transfection reagent. Twenty-four hours after transfection, the cells were collected and fluorescence was observed with a Nikon Diaphot 300 microscope.

Analysis of DNA and Amino Acid Sequence. DNASTAR and Clustalx software was used to identify the open reading frames and the amino acid sequences of various GFPxm mutants.

GenBank Accession Nos. GFPxm, AY013824; GFPxm16, AY013825; GFPxm18, AY013826; GFPxm19, AY013827; GFPxm161, AF435427; GFPxm162, AF435428; GFPxm163, AF435429; GFPxm191uv, AF435430; GFPxm19uv, AF435431; OFPxm, AF435432; and ShG24, AF435433.

\section{Results}

Generation of GFP Mutants with Different Absorption and Emission Spectra. GFPxm mutants were generated after site-directed mutagenesis or DNA shuffling. After they were transferred into ER2566 and induced with IPTG at $37^{\circ} \mathrm{C}$ for $4 \mathrm{~h}$, all mutants could be expressed to high levels and amount to as much as $32 \%$ of total cellular protein (data not shown). The fluorescence characteristics and ab- sorption and emission spectra of the mutants are summarized in Table 1 and Figure 1.

GFPxm Mutants with Enhanced Fluorescence at Higher Temperature. For most applications, it would be desirable if GFPs were highly expressed and correctly folded at $37^{\circ} \mathrm{C}$. However, the fluorescent wild-type GFPxm was obtained only after $24 \mathrm{~h}$ of expression at $15^{\circ} \mathrm{C}$, but no fluorescence was observed with expression at higher temperature despite a higher level of protein production. To overcome this problem, site-directed mutagenesis was performed at positions 64 to 66 of GFPxm, based on the results of GFP mutation (Cubitt et al., 1999). The mutagenesis produced two mutants, GFPxm18 and GFPxm19, with enhanced green fluorescence when expressed in $E$. coli at $37^{\circ} \mathrm{C}$ and a very small shift of excitation and emission maxima (Figure 1A and Table 1).

Mutants with UV-Excited Peak. As shown in Figure 1C and D, mutants GFPxm191uv, GFPxm19uv, and GFPxm181uv had two excitation peaks. GFPxm191uv had an emission peak at 510 $\mathrm{nm}$ and a major excitation peak at $498 \mathrm{~nm}$ in which fluorescence intensity was about two times higher than the minor excitation peak (394 nm). The spectra of GFPxm181uv were similar to that of GFPxm19uv but with lower fluorescence intensity. 


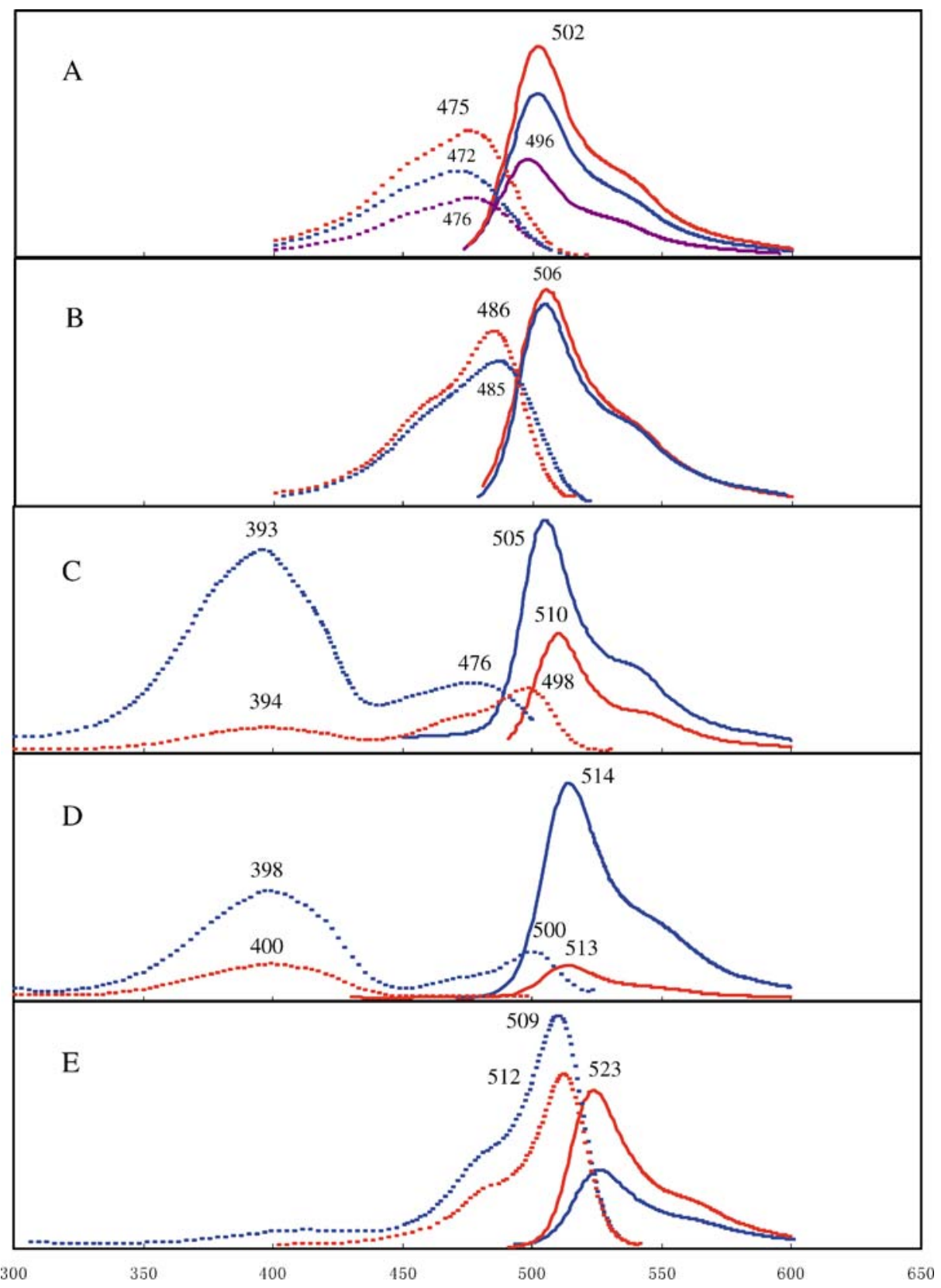

Fig. 1. Excitation-emission spectra for GFPxm mutants. Spectra for the wild-type GFPxm are shown for comparison. Within each pair of lines the excitation spectrum is the one at shorter wavelengths (dotted lines) and the emission spectrum is the one at longer wavelengths (solid lines). (A) Spectra of wild-type GFPxm (grey thick lines), GFPxm18 (black thick lines), and GFPxm19 (thin lines). (B) Spectra of GFPxm16 (thick lines) and ShG24 (thin lines). (C) Spectra of GFPxm19uv (thick lines) and GFPxm191uv (thin lines). (D) Spectra of GFPxm18uv (thin lines) and GFPxm181uv(thick lines). (E) Spectra of OFPxm (thick lines) and GFPxm163 (thin lines).

GFPxm18uv was obtained after T203H substitution in GFPxm18. This mutant had a single excitation peak at $400 \mathrm{~nm}$ and an emission peak at $513 \mathrm{~nm}$.

Mutants with Red Shift Spectra. Seven mutants had red shifts in their spectra. Double mutation of F64L and S65G in GFPxm16 led to a small shift of excitation and emission maxima to $485 \mathrm{~nm}$ and $506 \mathrm{~nm}$, respectively. Another mutant,
ShG24, containing six amino acid substitutions produced by DNA shuffling had similar excitationemission spectra with GFPxm16 (Figure 1B). Both GFPxm16 and ShG24 could be produced efficiently at high temperature with enhanced fluorescence intensities. The longest emission maximum (525 nm) was obtained when $\mathrm{F}$ replaced $\mathrm{T}$ in position 203 of GFPxm16. This mutant, however, had low fluorescence intensity and was named GFPxm162. Mu- 


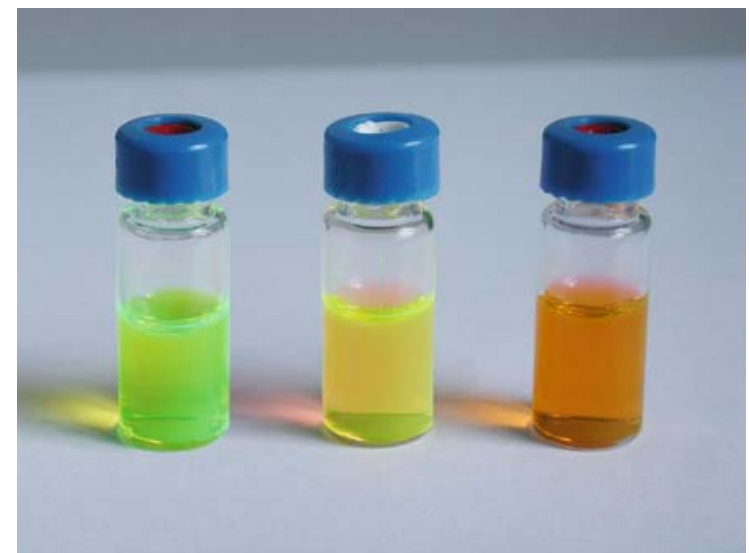

Fig. 2. Purified GFPxm18, GFPxm163, and OFPxm. GFP xm18 fluoresces green, GFPxm63 fluoresces yellow-green, and OFPxm fluoresces orange.

tant GFPxm163, with excitation peak at $512 \mathrm{~nm}$ and emission peak at $523 \mathrm{~nm}$, was the result of T203Y substitution in GFPxm16 (Figure 1E).

Mutant OFPxm was the result of T203Y substitution in ShG24. OFPxm and GFPxm163 had similar excitation-emission spectra (Figure 1E). It is interesting to note that although both mutants had similar spectra, GFPxm163 exhibited yellow-green fluorescence while OFPxm showed an orange color (Figure 2). Theoretically, wavelength at $523 \mathrm{~nm}$ belongs to the green spectrum. The yellow appearance of GFPxm163 was caused by an emission tail at longer wavelengths. OFPxm had the same emission peak at $523 \mathrm{~nm}$ and the tail at longer wavelengths, but its fluorescence was orange without a trace of green.

Effect of Expression Temperature on the Fluorescence Intensity of GFPxm Mutants. Figure 3 shows the normalized fluorescence intensity of five GFPxm mutants when expressed at different temperatures with EGFP and EYFP as controls (Lippincott-Schwartz et al., 2003). Our results showed that GFP fluorescence intensity decreased with increased temperature at expression. However, the fluorescence intensity of GFPxm16, GFPxm18, and GFPxm19 was higher than that of EGFP when the temperature was 25,32 , and $37^{\circ} \mathrm{C}$. GFPxm 18 had the strongest intensity in class 2 (Table 1, phenolate anion) at $37^{\circ} \mathrm{C}$, which was one and a half times higher than that of EGFP. When the temperature increased to $42^{\circ} \mathrm{C}$, GFPxm 16 had the strongest intensity in class 2 that was 2.3 times higher than that of EGFP. The fluorescence of OFPxm did not change significantly with the increasing of temperature, but the intensity was comparatively low (Figure 3A). In contrast, GFPxm163 had the strongest fluorescence intensity among all GFPxm mutants even expressed at $42^{\circ} \mathrm{C}$, but the intensity was lower than that of EYFP (Figure 3B).

Effects of Expression Time on the Fluorescence Intensity of GFPxm Mutants. ER2566 strains transformed with different GFPxm mutants were cultured at $37^{\circ} \mathrm{C}$ to an optical density of 0.2 to 0.3 . Protein expression levels in different mutants were similar (data not shown). After IPTG induction at $37^{\circ} \mathrm{C}$ for half an hour, GFPxm16, GFPxm18, and GFPxm19 showed high fluorescence intensity (Figure 4A). The fluorescence of GFPxm18 could even be observed by eye at this time. The fluorescence intensity of GFPxm 18 was about 1.6 times higher than that of EGFP at different time
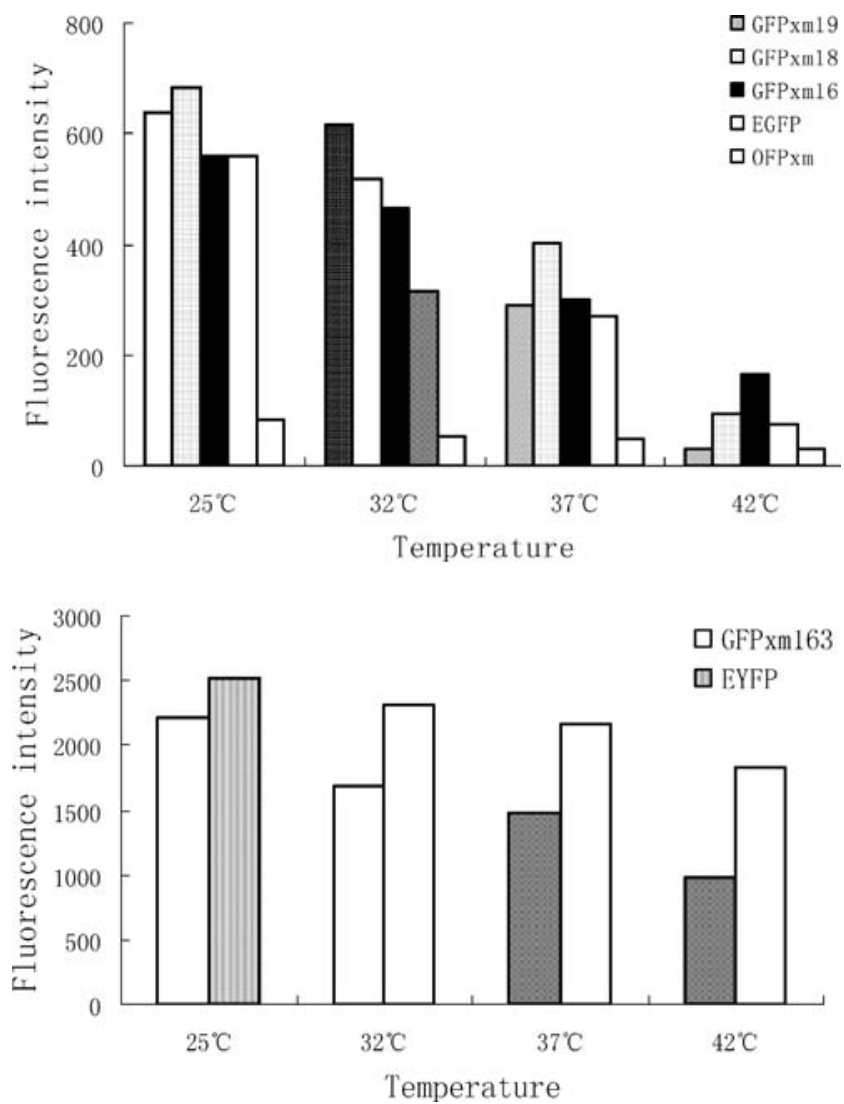

Fig. 3. Relative fluorescence intensity of wild-type and mutant GFPxms induced at different temperatures. ER2566 cells harboring plasmids encoding the wild-type or mutant GFPxm were induced with IPTG and incubated for $6 \mathrm{~h}$ at $25,32,37$, and $42^{\circ} \mathrm{C}$. The cell samples were diluted to give an OD value of 0.225 at a wavelength of 600 for determination of fluorescence in a Hitachi fluorescence spectrophotometer. GFP content of the cells was estimated by sodium dodecyl sulfate-polyacrylamide gel electrophoresis and expressed as fraction of total cellular protein. Relative fluorescence intensity was calculated as a ratio of fluorescence to fraction of GFP. 

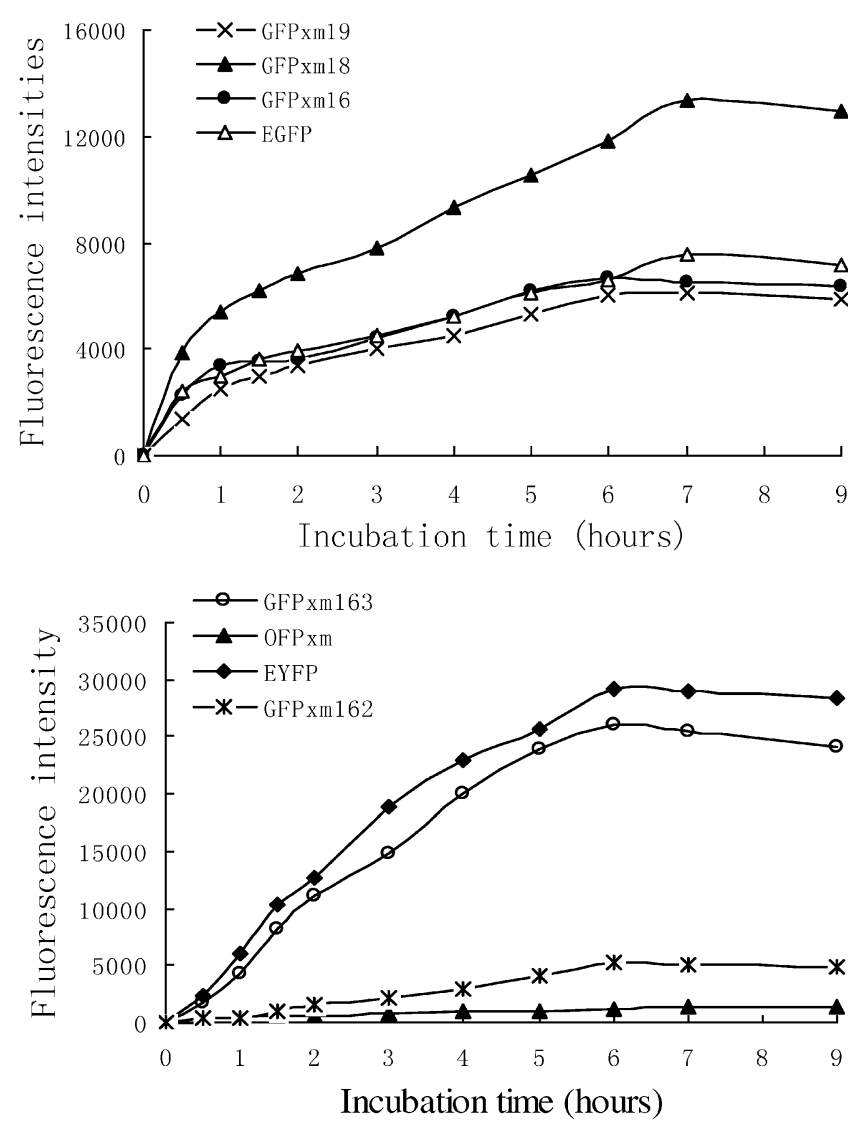

Fig. 4. Kinetics of induction of wild-type and mutant GF Pxm. ER2566 cells harboring plasmids encoding the wildtype or mutant GFPxm were induced with IPTG and incubated at $37^{\circ} \mathrm{C}$. Cell samples were taken at different times for determination of relative fluorescence intensity as described in the legend to Figure 3.

points, while GFPxm16 and GFPxm19 were almost equal to EGFP. The shapes of all intensity curves in Figure 4A are similar. The fluorescence intensity of GFPxm163 increased quickly with incubation time, but the intensity was slightly lower than that of EYFP (Figure 4B). The orange fluorescence fromOFPxm and GFPxm162 was not apparent until $4 \mathrm{~h}$ after induction but the fluorescence signal was still very weak. For all GFPxm mutants the fluorescence intensity reached a peak at 6 to $7 \mathrm{~h}$ after induction with IPTG at $37^{\circ} \mathrm{C}$.

Expression of GFPxm Mutants in Mammalian Cells. Six GFPxm mutants were cloned into mammalian expression vector pcDNA3.1 and the resultant plasmids were transfected into $\mathrm{CHO}$, Hela, and HepG2 cells. Mutants GFPxm16, GFPxm18, GFPxm19, and GFPxm163 could be successfully expressed in all of the three cell lines with fluorescence intensities comparable to that of EGFP. However, the fluorescence signal from GFPxm19uv was weaker and it was difficult to distinguish the fluorescence of OFPxm from a red background under green light illumination.

\section{Discussion}

In this study, 12 GFPxm mutants were generated by site-directed mutagenesis and DNA shuffling. The mutants showed shifted spectrum, enhanced fluorescence, or changed color compared to wild-type GFPxm. Most of the GFPxm mutants with high fluorescence intensity at $37^{\circ} \mathrm{C}$ resulted from replacement of bulky residues with smaller ones, such as the change of F64L, S65A, S65G, or Q69L. Similar results were also obtained with GFP (Tsien, 1998). As a marker in the application in living cells, GFPs should have the ability to fold correctly and produce fluorescence at high temperature. Our results showed that at least four variants can be well expressed at higher temperature and in several kinds of mammalian cells.

Tsien had divided the known GFP variants into seven classes on the distinctive component of their chromophores. These mutants of GFPxm can be divided into four classes of the seven (Table 1). The chromophores of GFPxm162, GFPxm163, and OFPxm belong to the class of phenolate anion with a stacked $\pi$-electron system. According to the mutagenesis studies on GFP and crystal structure of S65T GFP by Ormo et al. (1996), we postulate that the replacement of Ser at 65 by Gly promotes ionization of the chromophore, with an aromatic ring from aromatic residue at position 203 (Phe or Tyr) stacked next to the phenolate anion of the chromophore. The $\pi$-stacking interaction of the chromophore with aromatic ring causes the red shift of spectra.

OFPxm contains four different amino acids compared to GFPxm163. Three of them are localized at the $\mathrm{N}$-terminal of the protein and within the first $\beta$-strand, and belong to the same kind of amino acid as GFPxm163. Because mutation data from wild-type GFP showed that the change of residues in the $\mathrm{N}$-terminus of the protein would not cause a change of fluorescence signal, the mutation of these three residues in OFPxm should not be the reason for the fluorescence change. The fourth different residue in OFPxm is in position 69 and is near the chromophores site. Q in position 69 of GFPxm163 is a polar amino acid while $\mathrm{L}$ in position 69 of OFPxm is a hydrophobic amino acid. Therefore, it is possible that the residue difference in position 69 together with the same T203Y replacement leads to different microenvironments of the two chromophores and produces different colors. 
Several powerful fluorescent protein variants have been generated for efficient, selective optical labeling. Four photoactivable fluorescent proteins, PAGFP (Patterson and Lippincott-Schwartz, 2002), KFP (Chudakov et al., 2003), aceGFP-G222E (Gurskaya et al., 2003), and PA-mRFP1s (Verkhusha and Sorkin, 2005), can tag a fusion protein in living cells in a timed and spatially controlled fashion. Two photoswitchable variants, Kaede (Ando et al., 2002) and PS-CFP (Chudakov et al., 2004), can change their fluorescence color after intense illumination and have the advantage that they are also detectable, at a different wavelength, before photoconversion.

aceGFP-G222E is a mutant of a colourless green fluorescent protein homolog, acGFPL, from the nonfluorescent hydromedusa $A$. coerulescens. It can undergo UV light-induced photoconversion and reached a greater than 1000-fold increase of green fluorescence intensity (Gurskaya et al., 2003). Patterson and Lippincott-Schwartz found that substitution of histidine for threonine at position 203 of A. victoria GFP generated PA-GFP, which exhibits up to a 100-fold increase in green fluorescence excitation at $488 \mathrm{~nm}$ when illuminated with UV or violet light. Because the amino acid sequence identities of GFPxm from A. macrodactyla and acGFPL with A.victoria GFP are $84 \%$ and $92 \%$ respectively, some mutants from GFPxm can be engineered to have the exciting characters of aceGFP-G222E and PA-GFP in a similar fashion. In the present study, GFPxm18uv and GFPxm181uv had the same $\mathrm{T} 203 \mathrm{H}$ substitution and decreased absorbance at $476 \mathrm{~nm}$ with the expectation of photoactivation phenomena similar to that in PAGFP. We believe that they have the same photoactivation phenomena as PA-GFP.

\section{References}

Ando R, Hama H, Yamamoto-Hino M, Mizuno $\mathrm{H}$, Miyawaki A (2002) An optical marker based on the UV-induced green-to-red photoconversion of a fluorescent protein. Proc Natl Acad Sci USA 99, 12651-12656

Ando R, Mizuno H, Miyawaki A (2004) Regulated fast nucleocytoplasmic shuttling observed by reversible protein highlighting. Science 306, 1370-1373

Campbell RE, Tour O, Palmer AE, Steinbach PA, Baird GS, Zacharias DA, Tsien RY (2002) A monomeric red fluorescent protein. Proc Natl Acad Sci USA 99, 78777882

Chudakov DM, Belousov VV, Zaraisky AG, Novoselov VV, Staroverov DB, Zorov DB, Lukyanov S, Lukyanov KA (2003) Kindling fluorescent proteins for precise in vivo photolabeling. Nat Biotechnol 21, 191-194
Chudakov DM, Verkhusha VV, Staroverov DB, Souslova EA, Lukyanov S, Lukyanov KA (2004) Photoswitchable cyan fluorescent protein for protein tracking. Nat Biotechnol 22, 1435-1439

Crameri A, Whitehorn EA, Tate E, Stemmer WPC (1996) Improved green fluorescent protein by molecular evolution using DNA shuffling. Nat Biotechnol 14, 315-319

Cubitt AB, Woollenweber LA, Heim R (1999) Understanding structure-function relationships in the Aequorea victoria green fluorescent protein. Methods Cell Biol $58,19-30$

Gurskaya NG, Fradkov AF, Pounkova NI, Staroverov DB, Bulina ME, Yanushevich YG, Labas YA, Lukyanov S, Lukyanov KA (2003) A colourless green fluorescent protein homologue from the non-fluorescent hydromedusa Aequorea coerulescens and its fluorescent mutants. Biochem J 373(Pt 2), 403-408

Ito Y, Suzuki M, Husimi Y (1999) A novel mutant of green florescent protein with enhanced sensitivity for microanalysis at $488 \mathrm{~nm}$ excitation. Biochem Biophys Res Commun 264, 556-560

Karasawa S, Araki T, Yamamoto-Hino M, Miyawaki A (2003) A green-emitting fluorescent protein from Galaxeidae coral and its monomeric version for use in fluorescent labeling. J Biol Chem 278, 34167-34171

Labas YA, Gurskaya NG, Yanushevich YG, Fradkov AF, Lukyanov KA, Lukyanov SA, Matz MV (2002) Diversity and evolution of the green fluorescent protein family. Proc Natl Acad Sci USA 99, 4256-4261

Lippincott-Schwartz J, Patterson GH (2003) Development and use of fluorescent protein markers in living cells. Science 300, 87-91

Luo WX, Zhang J, Yang HJ, Li SW, Xie XY, Pang SQ, Li SJ, Xia NS (2000) Construction and application of an Escherichia coli high effective expression vector with an enhancer. Sheng Wu Gong Cheng Xue Bao 16, 578581

Ormo M, Cubitt AB, Kallio K, Gross LA, Tsien RY, Remington SJ (1996) Crystal structure of the Aequorea victoria green fluorescent protein. Science 273, 13921395

Patterson GH, Lippincott-Schwartz J (2002) A photoactivatable GFP for selective photolabeling of proteins and cells. Science 297, 1873-1877

Shaner NC, Campbell RE, Steinbach PA, Giepmans BN, Palmer AE, Tsien RY (2004) Improved monomeric red, orange and yellow fluorescent proteins derived from Discosoma sp. red fluorescent protein. Nat Biotechnol 22, 1567-1572

Tsien RY (1998) The green fluorescent protein. Annu Rev Biochem 67, 509-544

Verkhusha VV, Sorkin A (2005) Conversion of the monomeric red fluorescent protein into a photoactivatable probe. Chem Biol 12, 279-285

Xia NS, Luo WX, Zhang J, Xie XY, Yang HJ, Li SW, Chen M, Ng MH (2002) Bioluminescence of Aequorea macrodactyla, a common jellyfish species found in the east China sea. Mar Biotechnol 4, 155-162 